Berlinerin angehört, und das kann auch nicht eine Präsidentin allein. Dafür braucht es jede einzelne von Ihnen an ihrem Platz irgendwo in unserem ziemlich großen Land. djb ist da, wo Sie ihm gerade ein Gesicht geben. Und es freut uns sehr, dass dieses wichtige ehrenamtliche Wirken auf Landesebene zur Kenntnis genommen und gewürdigt wird. Es ist schön, dass unsere Vizepräsidentinnen solche Ehrungen erhalten, wie auch Ruth Schimmelpfeng-Schütte im Mai 2012, vor gut einem Jahr.

Liebe Renate, im Namen aller Kolleginnen im djb danke ich dir sehr herzlich dafür, dass du diese Aufgabe seit vielen Jahren mit großem Engagement wahrnimmst und dies sicher auch weiter tun wirst. Dass der djb in Bayern so bekannt ist, so stark ist, verdanken wir dir und den Kolleginnen im Landesverband, die ich jetzt nicht alle nennen kann. Aber es sei mir gestattet, die Vorsitzende der Regionalgruppe München Süd, Dr. Monika Zumstein, stellvertretend für alle besonders zu nennen, auch wenn dies heute in erster Linie dein Tag ist. Ich bin sicher, dass wir uns alle neidlos mit dir freuen. Herzlichen Glückwunsch, Renate. Und wenn du doch irgendwann mal was ausgefressen haben solltest, dann sag uns bitte rechtzeitig Bescheid, damit wir nicht denken, es gibt einen weiteren Orden.

\title{
Landesverband Berlin Sommerempfang am 12. Juni 2013
}

Direkt am Brandenburger Tor fand dieses Jahr am 12. Juni 2013 der traditionelle Sommerempfang des djb-Landesverbandes Berlin statt. Im Haus der Commerzbank trafen sich ca. 250 djb-Mitglieder und geladene Gäste aus Legislative, Politik und Gesellschaft - "alles, was Rang und Namen hat, ist heute Abend hier", begrüßte djb-Landesverbandsvorsitzende Prof. Asoc. Dr. Jutta Glock die Anwesenden.

Die Festrede hielt Dr. Sigrid Evelyn Nikutta, Vorstandsvorsitzende der Berliner Verkehrsbetriebe (BVG), die sich in ihrer Ansprache u.a. den Themen Entgeltgleichheit und Frauenquote widmete. Sie berichtete von den Gleichstellungsmaßnahmen der BVG und zeigte auf, mit welchen Maßnahmen die BVG gezielt versucht, den Anteil von Frauen in Führungspositionen zu erhöhen. So ist die Einhaltung einer bestimmten Frauenquote nicht nur allgemeines Unternehmensziel, sondern findet sich in allen Zielvereinbarungen für Abteilungsleiter wider, die damit auch ganz persönliche Anreize zu deren Umsetzung haben. Neben vielen weiteren inhaltlichen Punkten sorgte der von Nikutta zitierte Seminartitel „Protzen statt piepsen“ einer Fortbildungsveranstaltung für Frauen für hinreichend Gesprächs- und Diskussionsstoff, um einen schönen Sommerabend im angrenzenden Garten mit Blick auf das Brandenburger Tor ausklingen zu lassen.

\section{Festrede}

\section{Dr. Sigrid Evelyn Nikutta}

Vorstandsvorsitzende der Berliner Verkehrsbetriebe (BVG), Berlin

Sehr geehrte Frau Dr. Glock,

meine sehr verehrten Damen und, wenn auch wenigen, Herren,

vielen Dank für die Einladung zum Sommerempfang des Juristinnenbundes. Ich freue mich, heute zu Ihnen sprechen zu dürfen.

Meistens, wenn so viele Frauen vor mir sitzen, geht es um die Quote - deshalb ist es für mich eine willkommene Ab-

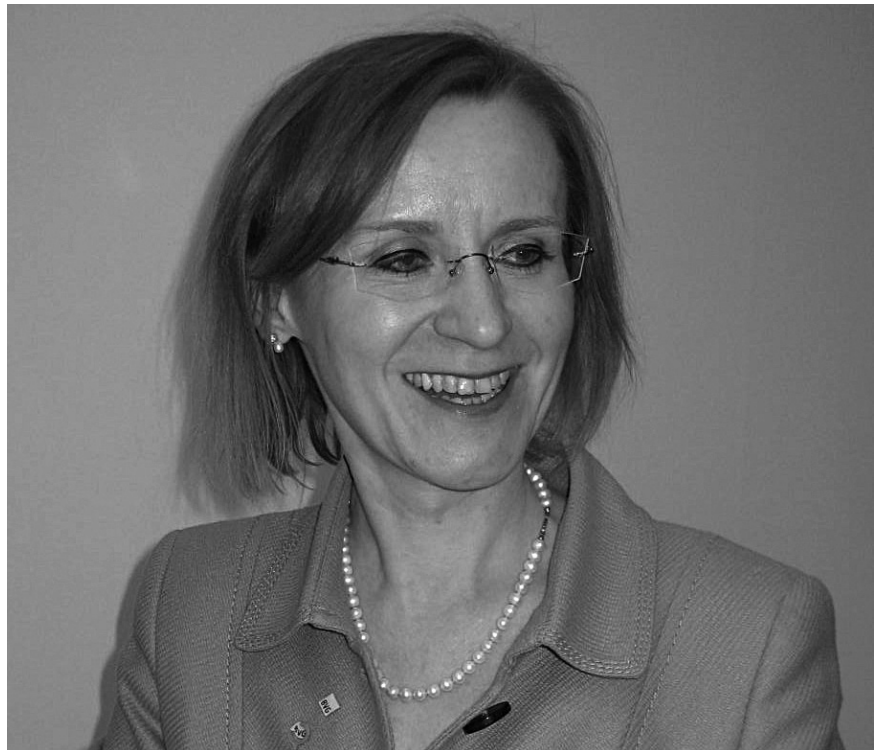

$\Delta$ Dr. Sigrid Evelyn Nikutta, Vorstandsvorsitzende der Berliner Verkehrsbetriebe (BVG), Berlin

wechslung, dass sie dieses Mal nicht im Vordergrund steht doch ganz werde ich auch diesmal nicht um sie herumkommen. Verstehen Sie mich bitte nicht falsch: Ich befürworte eine gesetzliche Regelung und setze mich dafür ein! Denn Selbstverpflichtungen der Unternehmen reichen nicht aus - Veränderungen passieren zu langsam! Die gläserne Decke besteht noch immer und erschwert den Aufstieg von Frauen.

Erst seit der Debatte über die Quote gibt es auch eine Debatte über Kompetenz - ginge es wirklich nach Kompetenz, dann gäbe es nicht so wenige Frauen in den Chefetagen: Frauen schließen beim Abitur und im Studium besser ab, und später findet man sie nicht mehr auf den gutbezahlten Posten - da läuft etwas falsch! Deshalb werden mit der Quote keine Ungerechtigkeiten geschaffen, wenn man nun auch Frauen für Leitungsfunktionen berücksichtigt, sondern bestehende Ungerechtigkeiten beseitigt! 
Nun zum eigentlichen, aber damit eng zusammenhängenden Thema meiner Rede: Der Entgeltungleichheit zwischen Männern und Frauen. Zweifelsohne werden Sie mir alle zustimmen, wenn ich sage: Tradierte Rollenmuster haben die monetäre Benachteiligung von Frauen über die Jahrhunderte zementiert. Doch was sind nun unsere Schlüsse daraus? In drei Abschnitten werde ich Ihnen meine Gedanken zu diesem Thema vortragen:

- Die aktuelle Situation und Gründe für Entgeltungleichheit.

- Wie können wir diese Ungerechtigkeit überwinden?

- Wie sieht es bei der BVG aus?

Ich beginne mit der aktuellen Situation und den Gründe für Entgeltungleichheit:

Die Entgeltlücke in Deutschland liegt seit Jahren unverändert bei 22 Prozent. Damit liegen wir auf dem vorletzten Platz in der Europäischen Union, wo Frauen im Durchschnitt 17 Prozent weniger verdienen. Fast ein viertel Jahr müssen deutsche Frauen durchschnittlich länger arbeiten, um auf das gleiche Gehalt wie Männer zu kommen. Der Equal Pay Day am 21. März markiert diesen Zeitraum, den Frauen über das Jahresende hinaus arbeiten müssen, um mit Männern gleichzuziehen.

Gemeinhin werden drei Gründe dafür ausgemacht:

1. Es gibt zu wenige Frauen in bestimmten Berufen, Branchen und in höheren Positionen.

2. Frauen unterbrechen oder reduzieren häufiger ihre Erwerbstätigkeit für die Kindererziehung und

3. die immer noch traditionell schlechtere Bewertung von typischen Frauenberufen.

Mit welchen Mitteln lässt sich gegensteuern und zur Entgeltgleichheit gelangen? Wie können wir diese Ungerechtigkeit überwinden? Um Frauen den Zugang zu gut bezahlten Positionen und Branchen zu ermöglichen, erfordert es den Mut der Wirtschaft - sie muss einsehen, dass es die Mischung macht und kein Potential verschenkt werden darf. Und es erfordert den Mut der Politik, endlich die gesetzlichen Rahmenbedingungen zu schaffen und die Wirtschaft in die Pflicht zu nehmen. Schließlich erfordert es den Mut der Frauen, in Männerdomänen einzudringen und sich Leitungspositionen zuzutrauen. $\mathrm{Zu}$ alledem müssen die Arbeitsbedingungen stimmen, um Frauen die Rückkehr nach der Elternzeit zu erleichtern: Aufstocken der Arbeitszeit nach der Teilzeitphase muss möglich und rechtlich verbindlich sein. Dadurch würde man auch erreichen, dass es für Männer attraktiver wird, Elternzeit zu nehmen. Ebenso wichtig sind faire Einkommen in Berufen, in denen hauptsächlich Frauen arbeiten - zum Beispiel Gesundheitsberufen, in denen 80 Prozent Frauen tätig sind. Über lange Zeit war es gesellschaftlich akzeptiert, und ist es bis heute, in diesen frauentypischen Berufen weniger zu zahlen. Doch die hohe fachliche und soziale Kompetenz etwa von Frauen in Gesundheitsberufen muss angemessen entlohnt werden. Dies rechtfertigt eine kritische Betrachtung, denn Bewertungssysteme wurden Jahrhunderte von Männern erstellt!

Wie sieht es nun bei der BVG aus? Lassen Sie mich zu meinem Unternehmen kommen und sogleich anmerken: Traditio- nell bedingt sind wir noch ein sehr männliches Unternehmen. Ende 2012 lag der Frauenanteil von den 13.000 Mitarbeiterinnen und Mitarbeitern der BVG bei 17,5 Prozent - und hier stagnieren wir schon seit einer ganzen Weile (seit 1995 unverändert). Deshalb haben wir uns als BVG-Vorstand die gezielte Förderung von Frauen zum Ziel gesetzt. Ich habe dabei das große Glück, dass ich mit meinen beiden Vorstandskollegen auf einer Linie bin, und so haben wir Ende letzten Jahres eine ambitionierte und verbindliche Frauenquote beschlossen: Zehn Prozent Steigerung in zehn Jahren! Bis 2017 soll der Frauenanteil auf 20 Prozent steigen, bis 2022 dann auf 27 Prozent. Und hierbei handelt es sich um keine abstrakte betriebliche Kennzahl - wir haben uns tatsächlich Abteilung für Abteilung angeschaut und die zu tätigenden Neueinstellungen bis 2022. Dann haben wir festgelegt:

- 50\% aller Stellen von ausscheidenden Männern, die wiederbesetzt werden, müssen mit Frauen neu besetzt werden.

- Außerdem müssen alle Frauen, die in Rente gehen, durch Frauen ersetzt werden.

- In den Bereichen, in denen bereits 50 Prozent oder mehr Frauen beschäftigt sind, muss der Frauenanteil nicht erhöht werden.

Gleichzeitig arbeiten wir eng mit unserer Gesamtfrauenvertretung zusammen, bieten extra Seminare für Frauen an, erleichtern Frauen mit flexiblen Arbeitszeitmodellen die Vereinbarkeit von Familie und Beruf und werben mit speziellen Kampagnen gezielt Mitarbeiterinnen und weibliche Auszubildende an. Und wir sind erfolgreich damit: 2012 haben wir für den gewerblichen Teil der Auszubildenden einen Anteil von 17 Prozent Mädchen eingestellt - im Vergleich: Alle Berliner Betriebe zusammen kommen durchschnittlich nur auf neun Prozent.

Wichtig war uns aber auch zu schauen, wie es beim Gehalt aussieht. Mitte 2012 haben wir die beiden Expertinnen für Entgeltgleichheit Frau Dr. Tondorf und Frau Dr. JochmannDöll beauftragt, unseren Tarifvertrag samt Entgeltordnung zu prüfen. Uns wurde bescheinigt, dass alle Mitarbeiterinnen und Mitarbeiter in der gleichen Entgeltgruppe den gleichen Lohn und die gleichen Zulagen erhalten - es gibt also keine unmittelbare Diskriminierung bei uns. Doch auch auf mittelbare Diskriminierung haben wir unsere Entgeltordnung geprüft - ob also auch in der Praxis, und nicht nur auf dem Papier, kein Unterschied zwischen Frauen und Männern gemacht wird. Beide Expertinnen haben mittels des EG-Check-Verfahrens frauendominierte und männerdominierte Tätigkeiten gegenüber gestellt und geschaut, ob gleichwertige Arbeit auch gleich bezahlt wird. Und kamen zu dem Ergebnis: Bei der BVG besteht Entgeltgleichheit! Lassen Sie mich noch ein wenig erläutern:

Der EG-Check wurde z.B. für Fahrtätigkeiten, die für die BVG besonders wichtig sind und die fast 6.000 der $13.000 \mathrm{Be}-$ schäftigten im BVG-Konzern übernehmen, durchgeführt - das Ergebnis zeigte, dass Entgeltgleichheit gewahrt ist: Alle Straßenbahn-, U-Bahn- und Omnibusfahrer/innen sind bei uns in der gleichen Entgeltgruppe eingeordnet. Die Prüfung erfolgte auf Basis des Punktesystems des EG-Checks, wobei jede Tätigkeit nach vier Bewertungsfeldern eingestuft wird: 
- Wissen und Können,

- psychosoziale Kompetenzen,

- Verantwortung,

- körperliche Anforderungen.

Es wurde deutlich, Tarifvertrag TV-N und Entgeltordnung sind ein gerechtes und langjährig bewährtes Eingruppierungssystem. Gleiche und gleichwertige Arbeit wird bei uns also gleich bezahlt! Ich möchte hier betonen, dass die Rahmenbedingungen in Berlin auch wirklich gut sind und dass Frauen hier bessere Chancen haben. Nicht nur ich persönlich und andere Frauen in Führungspositionen verdanken ihre Chance sicherlich der Politik.

Die Entgeltlücke ist hier in Berlin mit 15 Prozent im Vergleich zum Bundesdurchschnitt von 22 Prozent niedrig. Trotz- dem gibt es hier und bundesweit quer durch alle Branchen noch genug zu tun, damit mehr Frauen in Führungspositionen kommen und damit sie auf jedem Qualifikationsniveau auch attraktive Jobs bekommen. Es bedarf der gemeinsamen Anstrengung von Politik, Wirtschaft und Frauenverbänden, damit Frauen die gleichen Zugangschancen haben, um in allen Branchen und auf allen Ebenen tätig zu werden.

Und ich sage auch allen Frauen, besonders den jungen: Nehmt, was Euch zusteht, traut Euch mehr zu und nehmt Eure Partner in die Pflicht. Die Partnerwahl ist ein wichtiger Aspekt - wenn das alles zusammen kommt, dann wird sich auch die Entgeltlücke schließen!

Nun freue ich mich auf die Diskussion und wünsche uns allen interessante Gespräche!

\section{Geburtstage}

(April bis Juni 2013)

\section{Jahre}

- Elisabeth Pieper

Rechtsanwältin

Berlin

\section{Jahre}

\author{
- Ursula Voskuhl \\ Ministerialdirektorin a.D. \\ Bonn
}

\section{Jahre}

- Inge Reisse

Richterin a.D.

Hamburg 